\title{
Tumor Histology as prognostic factor in Locally Advanced Cervical Cancer
}

David Cantú De León, MD PhD¹, Lenny Gallardo Alvarado, MD MSc ${ }^{1}$, Rebeca Ramírez MD MSc ${ }^{1}$, Delia Pérez-Montiel MD², Victor Dominguez MD¹, Erik Díaz MD¹, Pamela Landin MD¹, Anai Ortega MD¹, Salim Barquet MD MSc ${ }^{3}$

1. Clinical Research department 2. Department of Pathology 3. Department of Gynecologic Oncology

\section{Background}

Cervical cancer is a public health problem in low-middle income countries due to the high frequency of locally advanced disease (LACC) at diagnosis, accounting up to $70 \%$ of cases. Clinical staging continues to be the most important prognostic factor; others, such as hemoglobin level, platelet count and tumor histology have been evaluated; the results continue to be controversial.

\section{Purpose}

Evaluate tumor histology as prognostic factor in terms of disease-free survival (DFS) and overall survival (OS) in a retrospective cohort of patients with LACC in a cancer reference center.

\section{Methods}

Records of 1065 patients treated with concurrent chemoradiation at Instituto Nacional de Cancerología of Mexico with confirmed cervical cancer stages IB2-IVA (Fiagoovo were reviewed. A descriptive and comparative analysis was conducted. DFS and OS were calculated for each histology with the Kaplan-Meier method and compared with Log-rank test, results were considered statistically significant if $p<0.05$.

\section{Results}

From 2005 to 2014 a total of 1065 records were retrieved

\begin{tabular}{|l|l|l|}
\hline Clinical Stage & N $=1065$ & $\%$ \\
\hline IB2 & & 7.1 \\
IIA & 76 & 4.2 \\
IIB & 44 & 56.1 \\
IIIA & 597 & 2.5 \\
IIIB & 27 & 25.6 \\
IVA & 273 & 4.5 \\
\hline Histology & 48 & \\
\hline Squamous Cell Carcinoma (SCC) & 917 & 86.1 \\
Adenocarcinoma (AC) & 105 & 9.9 \\
Adeno Squamous Carcinoma (ASC) & 7 & 0.7 \\
Other & 36 & 3.4 \\
\hline Grade & & \\
\hline well-differentiated & & 2.3 \\
moderately differentiated & 778 & 73.1 \\
\hline poorly differentiated & 263 & 24.6 \\
\hline
\end{tabular}
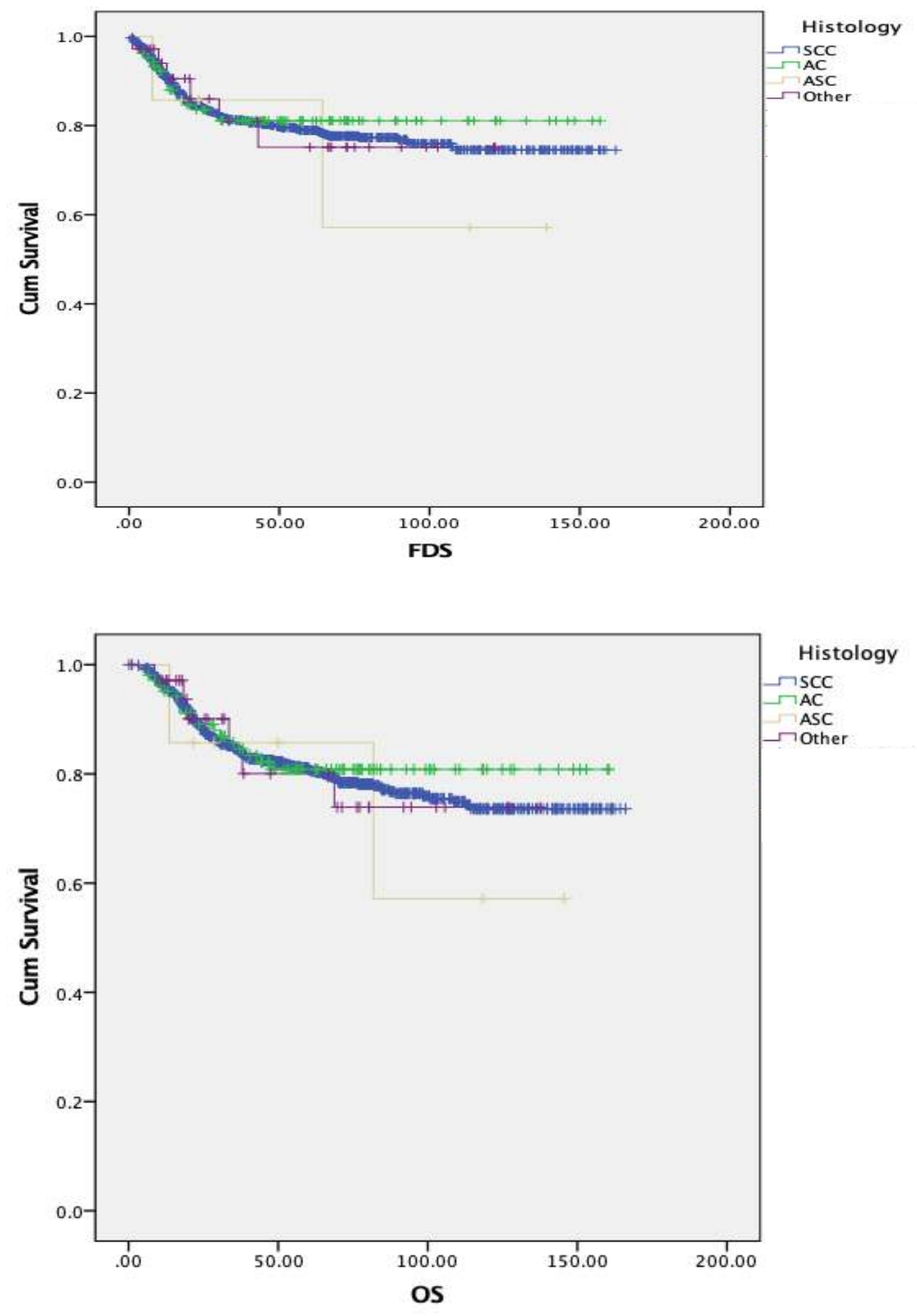

Mean Disease-free survival for SCC was 10 years, 9 for AC, and 6.9 for ASC, without a statistically difference $(p=0.365)$ Same findings occurred for OS, mean survival was 11 years, not finding impact of histology $(p=0.89)$.

Well differentiated tumors had OS of $100 \%$ at 5.8 years which was statistically better when compared with other grades $(p=0.032)$.

\section{Conclusions}

DFS and OS were comparable among different tumor histologies and was considerably better in well differentiated tumors, other factors should be considered. 